\title{
Plasma inorganic pyrophosphate and alkaline phosphatase in patients with pseudoxanthoma elasticum
}

\author{
Ana María Sánchez-Tévar ${ }^{1}$, María García-Fernández ${ }^{2}$, Belén Murcia-Casas ${ }^{3}$, José Rioja-Villodres ${ }^{1,4}$, \\ Juan Luis Carrillo ${ }^{3}$, Marta Camacho5, Matthias Van Gils ${ }^{6,7}$, Miguel Angel Sánchez-Chaparro ${ }^{1,3,4}$, \\ Olivier Vanakker ${ }^{6,7}$, Pedro Valdivielso ${ }^{1,3,4}$ \\ ${ }^{1}$ Lipid and Arteriosclerosis Laboratory, Centro de Investigaciones Médico-Sanitarias (CIMES), University of Málaga, Málaga, Spain; ${ }^{2}$ Department of \\ Physiology, Instituto de Investigación Biomédica de Málaga (IBIMA), University of Málaga, Málaga, Spain; ${ }^{3}$ Internal Medicine Unit, Hospital Virgen \\ de la Victoria, Málaga, Spain; ${ }^{4}$ Department of Medicine and Dermatology, Instituto de Investigación Biomédica de Málaga (IBIMA), University of \\ Málaga, Málaga, Spain; ${ }^{5}$ Obstetric and Gynecology Department, Hospital Virgen de la Victoria, Málaga, Spain; ${ }^{6}$ Center for Medical Genetics, Ghent \\ University Hospital, Ghent, Belgium; ${ }^{7}$ Department of Biomolecular Medicine, Ghent University, Ghent, Belgium \\ Contributions: (I) Conception and design: P Valdivielso, MA Sánchez-Chaparro; (II) Administrative support: AM Sánchez-Tévar; (III) Provision \\ of study materials or patients and controls: AM Sánchez-Tévar, B Murcia-Casas, JL Carrillo, M Camacho; (IV) Collection and assembly of data: \\ MA García-Fernández, J Rioja-Viollodres, M Van Gils; (V) Data analysis and interpretation: O Vanakker, M García-Fernández, P Valdivielso; \\ (VI) Manuscript writing: All authors; (VII) Final approval of manuscript: All authors. \\ Correspondence to: Miguel Angel Sánchez-Chaparro. Department of Medicine and Dermatology, University of Málaga. Boulevard Luis Pasteur 10, \\ 29071, Málaga, Spain. Email: masch@uma.es.
}

Background: Inorganic pyrophosphate (PPi) plays a major role inhibiting dystrophic calcification. The aim was to analyze levels of PPi in patients having pseudoxanthoma elasticum (PXE), and controls as well as the enzymes who regulate the PPi plasma concentration.

Methods: We collected fasting blood samples from PXE patients and age- and sex-matched controls in ethylenediamine tetraacetic acid (EDTA) and citrate-theophylline-adenosine-dipyridamole (CTAD) containing tubes. We measured PPi, ENPP1 mass and activity, alkaline phosphatase (AP) and tissue nonspecific alkaline phosphatase (TNAP), CD73 and Human Platelet Factor-4 (CXCL4).

Results: PPi in EDTA and CTAD samples were lower in PXE subjects than in controls $(1.11 \pm 0.26$ vs. $1.43 \pm 0.41 \mu \mathrm{M} / \mathrm{L}$ and $0.35 \pm 0.15$ vs. $0.61 \pm 0.18 \mu \mathrm{M} / \mathrm{L}$ respectively, $\mathrm{P}<0.05)$. TNAP and liver TNAP activities were also higher in PXE than in controls $(80.3 \pm 27.0$ vs. $63.3 \pm 16.4 \mathrm{UT} / \mathrm{L}$ and $25.6 \pm 14.9$ vs. $12.9 \pm 9.2 \mathrm{UT} / \mathrm{L}$ respectively, $\mathrm{P}<0.05)$. ENPP1 mass and activity as well as CD73 were almost identical. There was a weak but significant inverse correlation between TNAP activity and PPi levels (Pearson correlation $-0.379, \mathrm{P}<0.05$ ) in both groups.

Conclusions: High TNAP activity seems to contribute to low plasma levels of PPi in subjects with PXE, reinforcing the idea that pharmacological reduction of TNAP activity may help to reduce dystrophic calcification in PXE patients.

Keywords: Pseudoxanthoma elasticum (PXE); inorganic pyrophosphate (PPi); alkaline phosphatase (AP); nonspecific tissue alkaline phosphatase (TNAP); CD73; ENPP1; calcification

Submitted Sep 03, 2019. Accepted for publication Nov 29, 2019.

doi: $10.21037 /$ atm.2019.12.73

View this article at: http://dx.doi.org/10.21037/atm.2019.12.73 


\section{Introduction}

Inorganic pyrophosphate (PPi) plays a major role in preventing dystrophic calcification $(1,2)$. Plasma $\mathrm{PPi}$ is generated from nucleotides (ATP) by the ENPP1 enzyme. Lack of ENPP1 due to pathogenic variants in the ENPP1 gene, in both human and mouse, causes severe depletion of PPi and generalized arterial calcification of infancy (GACI) (3). Low levels of plasma PPi have been also reported in arterial calcification due to deficiency of CD73 (ACDC), caused by pathogenic variants in the 5'ectonucleotidase (NTSE) gene, which encodes a 5'Nucleotidase (CD73), an enzyme that converts extracellular adenosine monophosphate (AMP) into adenosine and inorganic phosphate (Pi) (4). Skin fibroblasts from ACDC patients have increased activity of tissue-non-specific alkaline phosphatase (TNAP), the major enzyme converting PPi into Pi (4).

Pseudoxanthoma elasticum (PXE) is a rare disease caused by pathogenic variants in the $A B C C 6$ gene, which is mainly expressed in the liver and the kidney. Clinically, PXE induces calcification and fragmentation of elastic fibers in the retina (neovascularization and a tendency for blindness), in the skin (papules and cutis laxa) and in the arterial wall (increased risk for peripheral artery disease and ischemic events).

The current pathogenesis of the disease has suggested that ABCC6 could be involved in ATP transportation from intracellular to the extracellular space. Therefore, in the light of this hypothesis, loss-of-function ABCC6 pathogenic variants would render less ATP available for ENPP1 and, consequently, less PPi is available (5).

Moreover, PPi is also a substrate for TNAP and it has been demonstrated in in vitro experiments that TNAP is significantly increased in PXE fibroblasts (6)

Two papers reported that plasma PPi levels are 30-40\% lower in PXE patients than in controls $(5,7)$. Mouse models of PXE reinforce the role of low PPi in the disease. In $A b c 6^{-1-}$ mice expressing human ENPP1, there was an elevation of plasma PPi and mineralization was reduced (8). Furthermore, oral administration of pyrophosphate inhibited connective tissue calcification (9).

The aim of this study was to analyze plasma levels of PPi and its association with the activities of serum ENPP1, 5'Nucleotidase and TNAP in subjects with PXE and in controls.

\section{Methods}

\section{Patients and controls}

Patients were recruited with an advertisement to the National Spanish Association for PXE. Patients were eligible when they fulfilled the clinical and molecular criteria for the diagnosis of PXE (typical skin and eye symptoms plus two pathogenic variants in the ABCC6 gene) (10). Age and sex-matched controls were recruited among healthy sanitary personnel. Neither patients nor controls were taking bisphosphonates. All participants signed informed consent. The Ethical Review Committee of the University Hospital of Málaga approved the study.

Demographic, anthropometric and clinical data were recorded. The severity of PXE was assessed using the Phenodex Score (11).

\section{Samples}

Nine-hour fasting whole blood was collected by intravenous puncture into serum, K2-EDTA, Citrate and citratetheophylline-adenosine-dipyridamole (CTAD) vacuum tubes (BD Vacutainer. Plymouth. UK). Blood samples were kept on ice and centrifuged, for $15 \mathrm{~min}$ at 3,000 rpm at $4{ }^{\circ} \mathrm{C}$. Serum (off the clot) and plasma aliquots were obtained on ice and stored at $-70{ }^{\circ} \mathrm{C}$ until assay.

CTAD blood tubes were centrifuged $15 \mathrm{~min}$ at 2,000 rpm at $4{ }^{\circ} \mathrm{C}$. Plasma was then transferred into separation Vivaspin 6 tubes 300,000 Molecular weight Cut off (Sartorius, ref: VS0652. Stonehouse. UK) and filter-centrifuged at $4,000 \mathrm{rpm}$ for $35 \mathrm{~min}$ at $4{ }^{\circ} \mathrm{C}$. Filtered plasma samples were stored at $-70^{\circ} \mathrm{C}$ until further processing. The ratio of charged CTAD plasma into vivaspin 6 tubes to eluted volume was considered for final calculations.

\section{PPi measurement}

PPi was measured in EDTA (ePPi) and CTAD (cPPi) plasma samples by an enzymatic commercial kit (Lonza, PPIlight Inorganic Pyrophosphate. Basel. Switzerland), as previously published $(12,13)$. Briefly, in a first reaction (R1) ATP sulfurylase converts PPi into ATP in the presence of an excess of adenosine 5'-phosphosulfate. In a second reaction (R2) ATP is degraded in the presence of 
luciferase yielding proportional luminescence. Reactions were displayed in a visible-clear 96 well microplate and luminescence (RLU) was measured in a Beckman DTX880 microplate reader (Beckman Coulter. California. USA). Sodium pyrophosphate tetrabasic (Sigma-Aldrich, ref: P8010, Saint Louis, USA) was used in $0.9 \% \mathrm{NaCl}$ as standard in concentrations of $10,5,2,1,0.5$ and $0.05 \mu \mathrm{M}$. Coefficient of variation $(\mathrm{CV})$ was $5.6 \%$.

\section{Enzymatic methods}

Alkaline phosphatase activity (ALP) was measured in serum samples by using two-reactive commercial assay containing diethanolamine $1 \mathrm{mmol} / \mathrm{L}$ and Magnesium Chlorine $0.5 \mathrm{mmol} / \mathrm{L}$ as buffer (R1). P-Nitrophenylphosphate was used as substrate (R2) (Spinreact, ref: 41242. Barcelona. Spain). Both R1 and R2 were mixed 4:1. The calibrator and internal quality controls were also purchased from the same manufacturer. The reaction was automated in a Mindray BS-380 discrete analyzer (MINDRAY, Shenzhen, China) according to the manufacturer's specifications. All samples were measured at once. $\mathrm{CV}$ was $3.9 \%$.

Tissue non-specific alkaline phosphatase activity (TNAP) was measured by the inhibition of the tissue specific ALP activity. In order to achieve this inhibition, $40 \mathrm{mmol} / \mathrm{L}$ phenylalanine (Sigma Aldrich, ref: P2126. St Louis. USA) was added to the reaction mixture (14) previously used to quantify the total ALP activity using the same automation. $\mathrm{CV}$ was also $3.9 \%$. Liver-TNAP and bone-TNAP isoenzymes activities were also quantified in serum. BoneTNAP precipitation was performed by the use of wheatgerm agglutinin as previously described (15). Briefly, $100 \mu \mathrm{L}$ of serum were added to $100 \mu \mathrm{L}$ of wheat-germ agglutinin (Ref L9640. Sigma Aldrich Chemie. Germany) precipitation mix $(5 \mathrm{~g} / \mathrm{L}$ of wheat-germ agglutinin and $2 \%$ triton $\mathrm{X}$ $100 \mathrm{v} / \mathrm{v}$ in $0.1 \mathrm{M}$ acetate buffer $\mathrm{pH} 4.5$ ). The mix was incubated for 30 minutes at room temperature and then centrifuged at $1,000 \times \mathrm{g}$ for $3 \mathrm{~min}$. The obtained supernatant contained the "residual" ALP/TNAP activity mainly composed of liver-TNAP and intestinal ALP activities. Liver-TNAP isoform activity from the supernatant was then directly measured by the addition of $40 \mathrm{mM}$ of Phe as previously described for TNAP activity measurements. Results were multiplied per two (because of the 1:1 sample dilution with the WGA precipitant). Finally, bone-TNAP isoform activity was calculated subtracting the above liverTNAP activity from serum TNAP activity.

ENPP1 activity was measured in serum samples by a kinetic enzymatic assay previously described (7). Briefly, $10 \mu \mathrm{L}$ of serum were incubated with $100 \mu \mathrm{L}$ of Thymidine 5'-monophosphate p-nitrophenyl ester sodium salt (Sigma Aldrich, ref: T4510. St. Louis. USA) $500 \mu \mathrm{mol} / \mathrm{L}$ in $100 \mathrm{mmol} / \mathrm{L}$ TRIS-HCl $\mathrm{pH} 8.9$, at $37^{\circ} \mathrm{C}$. Readings were assessed each $5 \mathrm{~min}$ for $1 \mathrm{~h}$ (12 cycles). Slopes were calculated (O.D. units/min) and then converted to $\mu \mathrm{U} / \mathrm{L}$ using a molar extinction coefficient of $18.4 \times$ $10^{-6} \mathrm{M}^{-1} \mathrm{~cm}^{-1}$. All reactions for ENPP1 activity were performed in duplicate in a 96 well visible-clear microplate, and measurements were displayed in a Beckman DTX880 multiplate reader. $\mathrm{CV}$ was $1.9 \%$. The specific activity of ENPP1 was calculated and expressed as $\eta \mathrm{U} / \eta \mathrm{g}$.

CD73 activity was measured in serum samples by using a two-reactive commercial assay (Diazime, ref: 41242. California. USA). Calibrators and quality controls were also purchased from the same manufacturer. The assay was automatized in the former Mindray BS-380, being the CV for this assay $1.7 \%$.

\section{ELISA Methods}

Human Platelet Factor-4 (CXCL4) (Sigma-Aldrich, RAB0402. Saint Louis. USA), Osteocalcin (R\&D systems, IC1419P. Abingdon. UK) and ENPP1 mass (Biovendor, ref: RD191124200R. Brno. Czech Republic) were measured by sandwich ELISA-based commercial kits. Optical density was measured in all ELISA methods at $450 \mathrm{~nm}$ wavelength with reference correction at $620 \mathrm{~nm}$ in the former Beckman DTX880 microplate reader.

CV for CXCL4, Osteocalcin and ENPP1 mass was 9.9\%, $3.3 \%$, and $2.5 \%$, respectively.

\section{Statistical analyses}

Data were analyzed using SPSS 25.0 software (IBM). Data are shown as mean $\pm \mathrm{SD}$, median (interquartile range) or number (\%). To compare between groups, Student $t$-test, Mann-Whitney test or ANOVA were applied. Person's test was used to evaluate correlation between variables. A P value $<0.05$ was considered significant.

\section{Results}

Table 1 shows demographics and anthropometry of PXE patients $(n=18)$ and controls $(n=16)$, well matched by age and sex. PXE patients showed a median of the Phenodex score of 5.5 , the involvement of skin and eyes being more 
Table 1 Demographics and clinical features in PXE subjects and controls

\begin{tabular}{lcc}
\hline Characteristic & PXE $(\mathrm{n}=18)$ & Controls $(\mathrm{n}=16)$ \\
\hline Age, year & $47 \pm 13$ & $45 \pm 12$ \\
Women/men $(\mathrm{n})$ & $13 / 5$ & $12 / 4$ \\
Body weight $(\mathrm{Kg})$ & $68 \pm 11$ & $67 \pm 7.3$ \\
Body mass index $\left(\mathrm{kg} / \mathrm{m}^{2}\right)^{*}$ & $26.8 \pm 4.7$ & $23.7 \pm 2.3$ \\
Systolic blood pressure $(\mathrm{mmHg})$ & $129 \pm 20$ & $128 \pm 15$ \\
Diastolic blood pressure $(\mathrm{mmHg})$ & $77 \pm 7$ & $78 \pm 7$ \\
Ankle/brachial index ${ }^{\dagger}$ & & \\
Right & $0.90 \pm 0.19$ & \\
Left & $0.94 \pm 0.15$ & \\
$<0.9$ & $7[39]$ & \\
Phenodex score & & Not applicable \\
Skin & $3[2,3]$ & \\
Eye & $2[2,3]$ & \\
Gastrointestinal & $0[0,0]$ & \\
Vascular & $0[0,0.15]$ \\
Cardiac & $0[0,0]$ \\
Total phenodex & $5.5[4,6]$ & Not applicable \\
\hline
\end{tabular}

Data are shown as number [\%], mean \pm SD or median [interquartile range]. ${ }^{\dagger}$, normal ankle/brachial index are $>0.9$; values $<0.9$ are diagnostic of peripheral arterial disease; *, $\mathrm{P}<0.05$. PXE, pseudoxanthoma elasticum.

Table 2 Biochemical features in PXE subjects and controls

\begin{tabular}{lcc}
\hline Parameter & PXE $(n=18)$ & Controls $(n=16)$ \\
\hline ePPi $(\mu \mathrm{M})^{\star}$ & $1.11 \pm 0.26$ & $1.43 \pm 0.41$ \\
cPPi $(\mu \mathrm{M})^{\star}$ & $0.35 \pm 0.15$ & $0.61 \pm 0.18$ \\
ALP $(\mathrm{U} / \mathrm{L})^{\star}$ & $156 \pm 52$ & $124 \pm 33$ \\
TNAP $(\mathrm{U} / \mathrm{L})^{\star}$ & $80.3 \pm 27.0$ & $63.3 \pm 16.4$ \\
Liver TNAP $(\mathrm{U} / \mathrm{L})^{*}$ & $25.6 \pm 14.9$ & $12.9 \pm 9.2$ \\
Bone TNAP $(\mathrm{U} / \mathrm{L})$ & $54.7 \pm 19.9$ & $50.4 \pm 11.2$ \\
ENPP1 $(\mathrm{ng} / \mathrm{mL})$ & $20.9 \pm 2.7$ & $20.3 \pm 2.6$ \\
ENPP1 $(\mu \mathrm{U} / \mathrm{L})$ & $6.79 \pm 1.08$ & $7.20 \pm 1.15$ \\
ENPP1 $(\eta \mathrm{U} / \mathrm{\eta g})$ & $8.03 \pm 1.27$ & $8.51 \pm 1.36$ \\
5NTD $(\mathrm{U} / \mathrm{L})$ & $4.78 \pm 1.03$ & $4.63 \pm 0.78$ \\
CXCL4 $(\mu \mathrm{g} / \mathrm{mL})^{*}$ & $5.47 \pm 0.18$ & $4.92 \pm 0.43$ \\
Osteocalcin $(\eta \mathrm{g} / \mathrm{mL})$ & $36.0 \pm 30.6$ & $29.8 \pm 11.7$ \\
\hline
\end{tabular}

Data are showed as mean $\pm \mathrm{SD}$. ${ }^{*}, \mathrm{P}<0.05$. PPi, inorganic pyrophosphate. ePPi, PPi measured in EDTA samples; cPPi, PPi measured in CTAD samples; ALP, alkaline phosphatase; TNAP, tissue non-specific alkaline phosphatase; ENPP1, ectonucleotide pyrophosphatase/phosphodiesterase 1; 5NTD, 5'ectonucleotidase. prevalent compared to other clinical manifestations. Basic hematological and biochemistry metabolites were similar in both groups, including calcium and phosphorus (data not shown).

Table 2 shows that PPi levels were significantly lower in PXE patients than in controls $(\mathrm{P}<0.05)$; in contrast, ALP and TNAP activities were higher $(\mathrm{P}<0.05)$. LiverTNAP isoform was significantly higher in PXE than in controls $(\mathrm{P}<0.05)$, whereas bone TNAP was similar in both groups. Mass and activity of ENPP1, 5'Nucleotidase activity and blood levels of osteocalcin were almost identical in both groups. We analyzed cPPi according to sex and menopausal status and we did not observe any significant differences between pre- and postmenopausal women and men $(0.44 \pm 0.16$ vs. $0.50 \pm 0.20$ vs. $0.48 \pm 0.29$ respectively, $\mathrm{NS})$. Same results were obtained in controls $(0.53 \pm 0.08 v \mathrm{~s}$. $0.65 \pm 0.14 v s .0 .74 \pm 0.3$, NS) than in PXE patients $(0.35 \pm 0.19$ vs. $0.39 \pm 0.17$ vs. $0.29 \pm 0.04$, NS). Main data are also summarized in Figure 1.

Furthermore, there was a significant inverse correlation between TNAP activity and cPPi levels (Pearson's coefficient $-0.379, \mathrm{P}=0.029)$ when both groups were considered as a whole (Figure 2). If we analyzed this correlation in controls and PXE groups we found also a negative correlation between TNAP and cPPi (-0.262 and -0.124 respectively, NS due to small sample size).

\section{Discussion}

Our results confirm previous reports $(5,7)$ showing that PPi levels are reduced in PXE patients when they were compared with age- and sex-matched controls. Measurements of CXCL4 values confirm that changes in PPi levels cannot be related to platelet degranulation $(16,17)$. CXCL4, a marker of platelet degranulation, was $11 \%$ higher in PXE than in controls and therefore platelets degranulation cannot be the cause of reduced PPi availability. Moreover, degranulation could be responsible of ATP release from platelets. ATP can be hydrolyzed by ENPP1/NT5E and by TNAP, thus increasing PPi levels. Since, the activity of ENPP1 and NT5E is not modified in PXE, changes could be related to the increased TNAP activity observed in PXE. However, it has been demonstrated that TNAP hydrolyze ATP at a very low rate compared to ENPP1 and NT5E (4). Reduced PPi levels measured in PXE could suggest that TNAP could also contribute to $\mathrm{PPi}$ degradation in addition to PPi 

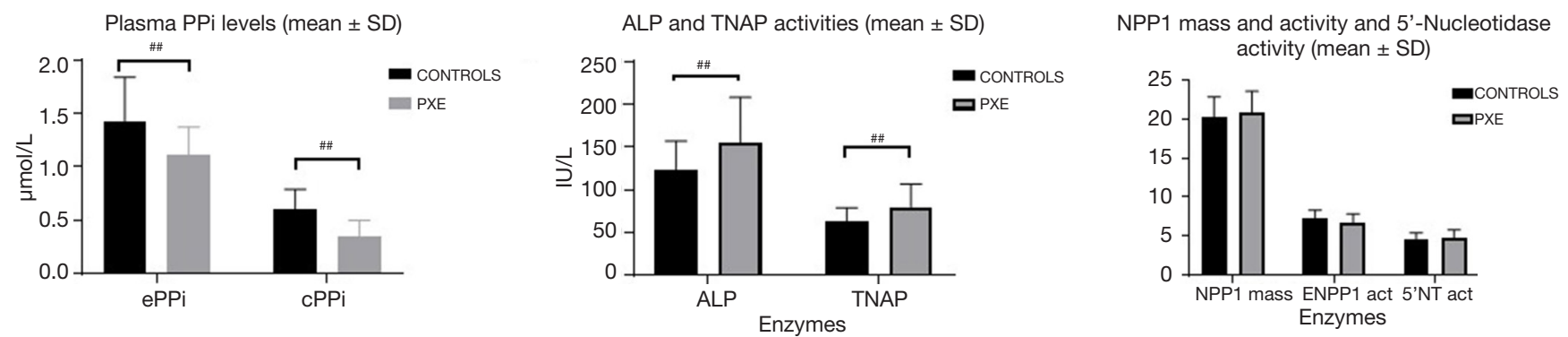

Figure 1 Plasma levels of PPi, Alkaline phosphatase and tissue-non specific alkaline phosphatase, ENPP1 activity and mass, and 5'Nucleotidase activity, in PXE patients and controls. ${ }^{\prime \prime}, \mathrm{P}<0.05$. PPi, inorganic pyrophosphate; ePPi, ppi measured in EDTA samples; cPPi, PPi measured in CTAD samples; ALP, alkaline phosphatase activity; TNAP, tissue non-specific alkaline phosphatase; ENPP1, ectonucleotide pyrophosphatase/phosphodiesterase 1; PXE, pseudoxanthoma elasticum.

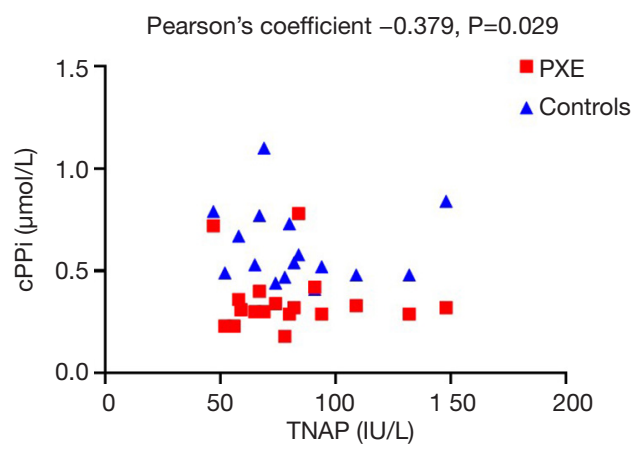

Figure 2 Pearson's coefficient between TNAP activity and cPPi both controls (blue square) and PXE (red triangles) groups. PXE, pseudoxanthoma elasticum; cPPi, inorganic pyrophosphate measured in CTAD samples; TNAP, tissue non-specific alkaline phosphatase.

production.

Interestingly, in PXE, reduction in PPi does not seem to be dependent on ENPP1 protein expression or activity, but more likely to increased activity of ALP/TNAP that hydrolyzes PPi releasing Pi. However, circulating levels of $\mathrm{Pi}$ are within normal range, possibly due to immediate capture of this ion within mineral deposits. These data are partly in agreement with those reported by Kauffenstein et al. (7), who showed in PXE reduced PPi and similar ENPP1 activity, but increased CD73 activity and no differences in ALP activity compared to controls. Despite of this, ALP was slightly higher in PXE than in controls $(\mathrm{P}=0.1)$; differences in both studies might be related with different methodology used in the measurement to ALP.

The present study failed to demonstrate changes in CD73 activity. CD73 activity induces AMP hydrolysis with release of Pi and adenosine that is known to inhibit TNAP (7). Therefore, in the absence of increased CD73, higher TNAP activity may be due to still unknown circulating factors (18) or to changes in energy metabolism through altered ROS signaling (19).

Serum of PXE patients showed higher ALP and TNAP activities compared to controls, even though values were in both cases within normal range. However, in the presence of lower PPi levels, even a small increase in ALP/TNAP activities may further contribute to reduce the inhibitory activity of PPi and this altered balance, in a long perspective, can significantly contribute to ectopic calcification $(20,21)$.

Since TNAP comprises different isoenzymes being expressed in liver, kidney (the two organs that most express ABCC6) and bone, we have investigated the activity of different TNAP isoforms in PXE and in controls. In serum, bone and liver TNAP are the most abundant isoforms. The TNAP percentage from the total ALP is method dependent and may vary from $90 \%(14,22)$ using an AMPTNAP based method, to $45 \%$ by the use of a DEA-Phe based method (14). In our study, we have used a DEA-Phe method and consistently, TNAP activity is approximately $50 \%$ from total ALP activity in PXE patients and controls respectively.

In our study, the liver TNAP isoform was higher in PXE than in controls (25.5 in PXE vs. 12.9 in controls, $\mathrm{P}<0.05)$. ALP and TNAP are involved in the inflammatory response as acute phase reactant (23), but in our study, PXE and controls had the same levels of serum C-reactive protein (data not shown) and were free of any kind of liver disease. It has been reported in a recent review that cellular signaling is disturbed in PXE fibroblast, with involvement of TFG- $\beta$, bone morphogenetic proteins (BMPs) and Pi (24). 
PXE murine cells also showed a marked increase of MPS2SMADs-RUNX2 pathways (25) and it is also known that RUNX2 is a transcription factor who increased expression of TNAP (22).

The clinical relevance of the negative association of PPi and TNAP seen in our study is unknown; however, although weak (Pearson's $\mathrm{R}-0.379, \mathrm{P}<0.05$, Figure 2) is consistent with the biological effect of TNAP and may help to focus on TNAP inhibitors as a therapeutic target in the treatment of PXE.

The absence of differences in bone-TNAP activity and osteocalcin concentration is consistent with a normal bone turnover in both PXE and controls.

Our findings might be of interest by several reasons: elevated TNAP activity may contribute to the lower levels of PPi shown in the disease and, therefore, to the calcification of elastic fibers, the hallmark of the disease. Furthermore, in PXE we have also found a negative correlation between PPi levels when measured in plasma CTAD and TNAP activity (Figure 1). However, negative correlation is necessary, but not sufficient to cause calcification, since a threshold should be reached to favor the development of ectopic calcification.

Nevertheless, inhibition of TNAP has been proposed as a target in PXE patients to counteract ectopic mineralization in vitro (6). In addition, it was used successfully in an $A b c c 6^{-1-}$ mouse model $(26,27)$. Very recently, it has been published that some compounds, orally active, with high activity against TNAP, can be entered in clinical trials in humans (28). In addition, TNAP expression can be downregulated through epigenetic modulation by apabetalone (29).

In conclusion, our study confirms that PXE patients have lower plasma levels of PPi coupled with an elevation in TNAP activity when compared to controls, which reinforces the research for drugs either inducing PPi and/or blocking TNAP as therapeutic target(s) for the disease.

\section{Acknowledgments}

The authors thank to the Spanish National Association of PXE patients. To the COST-Action 11615.

Funding: This study was supported by a grant (number PI16/01579) from the Fondo de Investigaciones Sanitarias (FIS), ISCIII, and funds from Grupo CTS-159, PAIDI and Plan de Empleo Juvenil (Ana María Sánchez-Tévar). M Van Gils is supported by a BOF research fellowship from the Ghent University. O Vanakker is a Senior Clinical Investigator of the research Foundation-Flanders (Belgium).

\section{Footnote}

Conflicts of Interest: The authors have no conflicts of interest to declare.

Ethical Statement: The authors are accountable for all aspects of the work in ensuring that questions related to the accuracy or integrity of any part of the work are appropriately investigated and resolved. Committee of the University Hospital of Málaga approved the study. All participants signed informed consent.

\section{References}

1. Orriss IR, Arnett TR, Russell RGG. Pyrophosphate: A key inhibitor of mineralisation. Curr Opin Pharmacol 2016;28:57-68.

2. Bäck M, Aranyi T, Cancela ML, et al. Endogenous Calcification Inhibitors in the Prevention of Vascular Calcification: A Consensus Statement From the COST Action EuroSoftCalcNet. Front Cardiovasc Med 2019;5:196.

3. Nitschke Y, Rutsch F. Inherited Arterial Calcification Syndromes: Etiologies and Treatment Concepts. Curr Osteoporos Rep 2017;15:255-70.

4. Jin H, St Hilaire C, Huang Y, et al. Increased activity of TNAP compensates for reduced adenosine production and promotes ectopic calcification in the genetic disease ACDC. Sci Signal 2016;9:ra121.

5. Jansen RS, Duijst S, Mahakena S, et al. ABCC6-mediated ATP secretion by the liver is the main source of the mineralization inhibitor inorganic pyrophosphate in the systemic circulation - Brief report. Arterioscler Thromb Vasc Biol 2014;34:1985-9.

6. Boraldi F, Annovi G, Bartolomeo A, et al. Fibroblasts from patients affected by Pseudoxanthoma elasticum exhibit an altered PPi metabolism and are more responsive to procalcifying stimuli. J Dermatol Sci 2014;74:72-80.

7. Kauffenstein G, Yegutkin GG, Khiati S, et al. Alteration of Extracellular Nucleotide Metabolism in Pseudoxanthoma Elasticum. J Invest Dermatol 2018;138:1862-70.

8. Zhao J, Kingman J, Sundberg JP, et al. Plasma PPi Deficiency Is the Major, but Not the Exclusive, Cause of Ectopic Mineralization in an Abcc6-/- Mouse Model of PXE. J Invest Dermatol 2017;137:2336-43.

9. Dedinszki D, Szeri F, Kozák E, et al. Oral administration of pyrophosphate inhibits connective tissue calcification. 
EMBO Mol Med 2017;9:1463-70.

10. Plomp AS, Toonstra J, Bergen AAB, et al. Proposal for updating the pseudoxanthoma elasticum classification system and a review of the clinical findings. Am J Med Genet Part A 2010;152A:1049-58.

11. Pfendner EG, Vanakker OM, Terry SF, et al. Mutation detection in the ABCC6 gene and genotypephenotype analysis in a large international case series affected by pseudoxanthoma elasticum. J Med Genet 2007;44:621-8.

12. Eriksson J, Karamohamed S, Nyrén P. Method for RealTime Detection of Inorganic Pyrophosphatase Activity. Anal Biochem 2001;293:67-70.

13. Ghetta A, Matus-Ortega M, García-Mena J, et al. Polynucleotide phosphorylase-based photometric assay for inorganic phosphate. Anal Biochem 2004;327:209-14.

14. Matsushita M, Irino T, Kamiyama K, et al. Evaluation of a method for measuring tissue non-specific alkaline phosphatase activity in healthy subjects. Ann Clin Biochem 2007;44:544-8.

15. Day AP, Saward S, Royle CM, et al. Evaluation of two new methods for routine measurement of alkaline phosphatase isoenzymes. J Clin Pathol 1992;45:68-71.

16. George JN, Thoi LL, Morgan RK. Quantitative analysis of platelet membrane glycoproteins: Effect of platelet washing procedures and isolation of platelet density subpopulations. Thromb Res 1981;23:69-77.

17. Tolouian R, Connery SM, O’Neill WC, et al. Using a filtration technique to isolate platelet free plasma for assaying pyrophosphate. Clin Lab 2012;58:1129-34.

18. Lomashvili KA, Garg P, Narisawa S, et al. Upregulation of alkaline phosphatase and pyrophosphate hydrolysis: Potential mechanism for uremic vascular calcification. Kidney Int 2008;73:1024-30.

19. Demer LL, Tintut Y. Vascular Calcification. Circulation 2008;117:2938-48.

20. Boraldi F, Annovi G, Vermeer C, et al. Matrix Gla Protein and Alkaline Phosphatase Are Differently Modulated

Cite this article as: Sánchez-Tévar AM, García-Fernández M, Murcia-Casas B, Rioja-Villodres J, Carrillo JL, Camacho M, Van Gils M, Sánchez-Chaparro MA, Vanakker O, Valdivielso P. Plasma inorganic pyrophosphate and alkaline phosphatase in patients with pseudoxanthoma elasticum. Ann Transl Med 2019;7(24):798. doi: 10.21037/atm.2019.12.73 in Human Dermal Fibroblasts from PXE Patients and Controls. J Invest Dermatol 2013;133:946-54.

21. Boraldi F, Bartolomeo A, Li Q, et al. Changes in dermal fibroblasts from Abcc6(-/-) mice are present before and after the onset of ectopic tissue mineralization. J Invest Dermatol 2014;134:1855-61.

22. Haarhaus M, Brandenburg V, Kalantar-Zadeh K, et al. Alkaline phosphatase: a novel treatment target for cardiovascular disease in CKD. Nat Rev Nephrol 2017;13:429-42.

23. Pike AF, Kramer NI, Blaauboer BJ, et al. A novel hypothesis for an alkaline phosphatase "rescue" mechanism in the hepatic acute phase immune response. Biochim Biophys Acta 2013;1832:2044-56.

24. Van Gils M, Nollet L, Verly E, et al. Cellular signaling in pseudoxanthoma elasticum: an update. Cell Signal 2019;55:119-29.

25. Hosen MJ, Coucke PJ, Le Saux O, et al. Perturbation of specific pro-mineralizing signalling pathways in human and murine pseudoxanthoma elasticum. Orphanet J Rare Dis 2014;9:66.

26. Ziegler SG, Ferreira CR, Macfarlane EG, et al. Ectopic calcification in pseudoxanthoma elasticum responds to inhibition of tissue-nonspecific alkaline phosphatase. Sci Transl Med 2017. doi: 10.1126/scitranslmed.aal1669.

27. Li Q, Huang J, Pinkerton AB, et al. Inhibition of TissueNonspecific Alkaline Phosphatase Attenuates Ectopic Mineralization in the Abcc6-/- Mouse Model of PXE but Not in the Enpp1 Mutant Mouse Models of GACI. J Invest Dermatol 2019;139:360-8.

28. Pinkerton AB, Sergienko E, Bravo Y, et al. Discovery of 5-((5-chloro-2-methoxyphenyl)sulfonamido)nicotinamide (SBI-425), a potent and orally bioavailable tissuenonspecific alkaline phosphatase (TNAP) inhibitor. Bioorg Med Chem Lett 2018;28:31-4.

29. Gilham D, Tsujikawa LM, Sarsons CD, et al. Apabetalone downregulates factors and pathways associated with vascular calcification. Atherosclerosis 2019;280:75-84. 